\title{
Going single, but not alone
}

By nature, most biological studies are averages over ensembles of cells or molecules. But averaging has its problems as it may mask important individual variations. To understand the molecular mechanisms underlying living systems, it will be necessary to complement such ensemble approaches with analyses of their individual units: single cells and single molecules. Biology needs more single-object approaches, and this will require overcoming substantial technical challenges.

Thanks to technological developments like automated sequencers, microarrays and powerful mass spectrometers, impressive genomics and proteomics efforts have brought within reach the compilation of an exhaustive inventory of macromolecules in living systems. Capitalizing on these lists of parts, functional 'omics' enterprises are identifying patterns in the behavior of the parts, such as gene-expression profiles, while systems biologists are mapping their interactions.

But elucidating some important mechanistic details will require the ability to analyze the composition of single cells, and to observe interactions and other processes both in single cells and using isolated single molecules in vitro. For this, biologists need tools for assessing presence, quantity, localization, movement and forces at the single-molecule level.

Take, for example, the process of actin-mediated transport. Nanoscale measurement of the movement of individual myosin proteins along actin in vitro shows that the proteins take discrete steps, rather than sliding smoothly along the fiber. Measuring the movement of an ensemble of myosin molecules, however, provides no clue as to this mechanism.

Similarly, single-cell observations bring insights, such as coincidence. It is one thing to know that two genes become expressed in response to a stimulus, but it is enlightening to understand whether the two genes are coinduced in the same cells or in different subpopulations of the cells. Furthermore, a small increase in expression measured at the population level may mean either a homogenous small increase across the population or a drastic increase in a subset of cells. Thus by analyzing single cells one also shifts from an analog to a digital mode of measurement, which is critical to derive mechanistic information.

What will it take to further the capacity for singlemolecule and single-cell studies? First and foremost, it is crucial to improve experimental sensitivity. Presently most single-molecule observations are made close to the limit of detection of the available assays.
Improving sensitivity and accuracy requires better probes. Fluorescence reagents have been at the core of single-cell and single-molecule detection, but considering their brightness, photostability, specificity and size, much more work is needed. The ideal probes, fluorescent or not, must be small, minimally perturb the biological system, and provide strong, stable and specific signals.

New instruments and molecular tools must also provide better signal amplification with minimal perturbation of the biology. Refining and increasing the availability of microfluidics devices, which allow dramatic reduction of sample volumes, will be central.

Another important aspect of single-object approaches is the capacity for multiple measurements. In biophysics, repeated observations are necessary to distinguish a real event from the noise. In single-cell approaches, biological noise is even more likely to confound observations: there is no way of inferring whether the cell you are observing is the odd one out or a true representation of the system. Thus, although it is important to go beyond population averaging, it is crucial to retain the capacity to perform population statistics. The answer most likely lies in miniaturization and highly parallel processing, with computational tools for data acquisition and deconvolution.

What the single-object challenge will require, really, is the convergence and integration of multiple disciplines. Biologists must join forces with biophysicists, and the creativity of physicists, chemists and engineers will be key to devising the necessary reagents and instruments. It is therefore crucial, now, to establish lines of communication between these communities.

The success of two conferences that made their debut in June is a very encouraging sign of such communication. The first Gordon Research Conference on "Single molecule approaches to biology", in New Hampshire, USA, saw one of the largest responses to a new Gordon conference. Although biophysicists in attendance outnumbered biologists, the meeting was a prime opportunity to discuss challenges and to foster collaborations. Similarly, the first "International workshop on approaches to single-cell analysis", at Uppsala University, in Sweden, gathered a diverse audience of biologists, chemists and engineers who were eager to collaborate. Given the success of these two meetings, the organizers have already made plans for their next editions. So stay tuned and mark your calendars. There is no reason for going single alone. 